\title{
CONSERVATION-ORIENTED HBIM. THE BIMEXPLORER WEB TOOL.
}

\author{
Ramona Quattrini ${ }^{\mathrm{a}}{ }^{*}$ Roberto Pierdicca ${ }^{\mathrm{a}}$, Christian Morbidoni $^{\mathrm{b}}$, Eva Savina Malinverni ${ }^{\mathrm{a}}$ \\ ${ }^{a}$ Universtitá Politecnica delle Marche, Dipartimento di Ingegneria Civile, Edile e dell' Architettura \\ Via Brecce Bianche, 60100, Ancona, Italy (r.quattrini, r.pierdicca, e.s.malinverni)@ univpm.it \\ ${ }^{a}$ Universtitá Politecnica delle Marche, Dipartimento di Ingegneria dell' Informazione \\ Via Brecce Bianche, 60100, Ancona, Italy (c.morbidoni)@univpm.it
}

\section{WG HBIM for management and maintenance}

KEY WORDS: Data Standardization, Taxonomies, Interoperability, Ontologies, Shared parameters, Representation workflow

\begin{abstract}
:
The application of $(\mathrm{H}) \mathrm{BIM}$ within the domain of Architectural Historical Heritage has huge potential that can be even exploited within the restoration domain. The work presents a novel approach to solve the widespread interoperability issue related to the data enrichment in BIM environment, by developing and testing a web tool based on a specific workflow experienced choosing as the case study a Romanic church in Portonovo, Ancona, Italy. Following the need to make the data, organized in a BIM environment, usable for the different actors involved in the restoration phase, we have created a pipeline that take advantage of BIM existing platforms and semantic-web technologies, enabling the end user to query a repository composed of semantically structured data. The pipeline of work consists in four major steps: i) modelling an ontology with the main information needs for the domain of interest, providing a data structure that can be leveraged to inform the data-enrichment phase and, later, to meaningfully query the data; ii) data enrichment, by creating a set of shared parameters reflecting the properties in our domain ontology; iii) structuring data in a machine-readable format (through a data conversion) to represent the domain (ontology) and analyse data of specific buildings respectively; iv) development of a demonstrative data exploration web application based on the faceted browsing paradigm and allowing to exploit both structured metadata and 3D visualization. The application can be configured by a domain expert to reflect a given domain ontology, and used by an operator to query and explore the data in a more efficient and reliable way. With the proposed solution the analysis of data can be reused together with the 3D model, providing the end-user with a non proprietary tool; in this way, the planned maintenance or the restoration project became more collaborative and interactive, optimizing the whole process of HBIM data collection.
\end{abstract}

\section{INTRODUCTION}

Building Information Modelling (BIM) has become a common standard tool that is used for the management of the whole life cycle of the construction activities. Even if the latter cannot be applied to the Cultural Heritage domain, the application of (H)BIM within the domain of Architectural Historical Heritage has huge potential that the research community is, nowadays, investigating. The final product of the HBIM process should be a model that, before being used as a professional tool, might be populated by attributes for making it suitable for engineering and conservation applications (this process can be defined as data enrichment phase). An enriched (H)BIM in the conservation/restoration domain can thus be exploited in a planned maintenance scenario, that is potentially endless. The brief literature review proposed in the next Section (2) demonstrates the huge potential of an HBIM approach applied to the domain of restoration/conservation of complex architectures (Achille et al., 2012). Notwithstanding, up to now, several obstacles are preventing HBIM towards a complete conversion in practical use. First of all, the modelling tools in the existing platforms allow simple operations only, that are not suitable to thoroughly represent the geometric irregularity of complex shapes. In this light, some issues of accuracy and data granularity are common (Apollonio et al., 2016). Further, in a $\mathrm{BIM}$-oriented approach the 3D models are a data collector populated by both geometrical and non-geometrical data which, in the Cultural Heritage $(\mathrm{CH})$ field, embody a large set of information related to various themes: historical documents, monitoring data,

\footnotetext{
${ }^{*}$ Corresponding author
}

structural information, conservation or restoration state and so on. Hence, the realization of a 3D model fully interoperable and rich in its informative content could represent a very important change towards the management of the conservation/restoration steps of an historical building. Is therefore evident the necessity of introducing, in the conservation chain, some tools which could make the process fully interoperable. Given the above, the following research question is becoming paramount: how this database can be interrogated, edited and upgraded avoiding, or at least minimizing, the issues of continuous conversions process caused by the difference of software between all the actors involved, enhancing a collaborative and transparent approach?

The work described in these pages presents a novel approach to solve this interoperability issue, by developing and testing a web tool based on the workflow, that will be detailed in Section 3 and here briefly represented in the Figure 2, experienced choosing as the case study a Romanic church in Portonovo, Ancona, Italy (Quattrini et al., 2015). The main objective of the proposed methodology is to take advantage from the Semantic Web paradigm, to allow the end user to perform advanced queries over an enriched HBIM model.

The "BIMexplorer" tool, designed for in-depth analysis, is conceived to allow non-expert users to perform semantic inquiries over the models and to visualize the results of their research directly on the browser. This can be very helpful especially in the case of monitoring the status of conservation of a historical building. The proposed solution in fact enable the practitioners to investigate over each single architectural component, getting pre- 


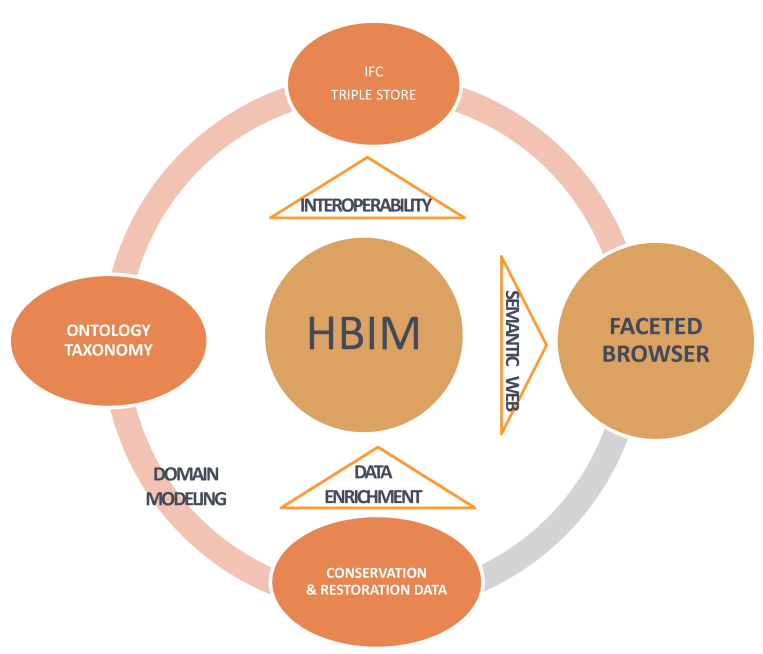

Figure 1. The comprehensive workflow for management of restoration and conservation data, allowing the interoperability between HBIM and faceted browser BIMExplorer thank to domain ontologies.

cise and reliable information about the presence of damages and their nature, the last year of inspection or link to pictures and 2D detailed drawings . It will be demonstrated in the Results Section (4) how such application can be configured by a domain expert to reflect a given domain ontology, and used by an operator to query and explore the data in a more efficient and reliable way. With the proposed solution, the planned maintenance or the restoration project became more collaborative and interactive, optimizing the whole process of HBIM data collection.

\section{RELATED WORKS}

Whether the classical use of BIM for new buildings is almost well established, the inherent characteristics of historical buildings like the uniqueness of components and the absence of the concept of life-cycle makes its applicability in the $\mathrm{CH}$ domain not exploited at all (Volk et al., 2014). In fact, an historical building is the product of a non-industrial process of construction and all the uses of a management tool are motivated by analysis, conservation and maintenance purposes (Figure ??). However, the use of (H)BIM is growing its interest within the researchers dealing with Cultural Heritage. The literature demonstrates how its use within this specific domain is becoming, nowadays, paramount (Apollonio et al., 2012). By using such technology for the management of the existing heritage allows one to exploit several benefits (Logothetis et al., 2015): remote reviewing of the building exterior and interior, surveying of different periods of time, estimation of the structure using images to understand texture, massing and form, enhancement of understanding the dynamics of a structure over time.

Its benefits have been broadly demonstrated also in the restoration domain ( $\mathrm{Li}$ et al., 2014). In the Italian Panorama for instance, the activities conducted on Milan Cathedral are noteworthy. In (Achille et al., 2012) the survey and model of the big spire to support restoration works is described. Also the work of (Ma et al., 2015) is important to be mentioned; the authors describe several aspects and requirements that should be considered when developing a BIM system for the built heritage for both on site and office work. Introducing BIM into the preservation and restoration of the timber frame of traditional historic

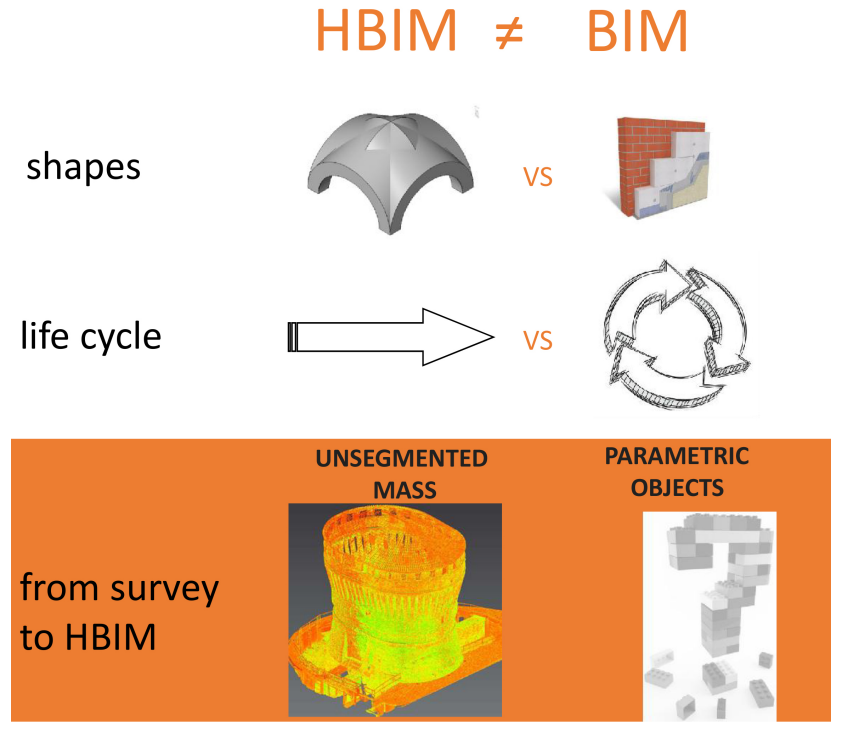

Figure 2. The image depicts the main differences between a BIM for the construction and the HBIM for the built heritage.

buildings in Taiwan, the establishment of visualization model, the design of parametric component objects and the update of the information for object restoration were carried out to increase the reuse of objects, demonstrating the improvement of the efficiency of restoration. Besides, in (Biagini et al., 2016), working on the SS. Nome di Maria church (Italy), an innovative approach to the construction management of historical building interventions, based on BIM technologies was proposed. Parametric modelling of historical buildings (starting with laser scanner survey), LoD (level of detail) definition, measurement accuracy, parametric modelling of the site and the several phases of restoration work, 3D graphic representation of safety procedures and related tools are discussed. In this regards, it is useful for the readers to make mention to the new regulation UNI UNI 11337$4: 2017^{1}$, now in force also in Italy. Specifically in the part 4 , the regulation standardizes the LOD for restoration purposes. It is not a coincidence that this regulation is up to an Italian commission, since the main building activities are oriented towards the built heritage that is spread and of high quality all over the country.

Nevertheless, even if the representation of ancient building plays a pivotal role for many purpose (Centofanti and Brusaporci, 2012) and especially for the restoration, in a (H)BIM oriented approach the data enrichment (and its related information management) represents the crucial part. Henceforth, the storage, the portability and the interoperability among architects and cultural heritage actors dealing with 3D technologies will represent the main research challenge. In fact, digital repositories seem unable to guarantee affordable features in the management of 3D models and their metadata (Felicetti and Lorenzini, 2011); this is mainly because of the nature of most of the available data format for 3D encoding, not suitable for the necessary portability required by $3 \mathrm{D}$ information across different systems. This issue generates the bottleneck that, even if having access to the information stored in a BIM model is essential (for instance to know the degree of conservation of a good), insiders and practitioners face with the problem of proprietary software which prevents the interoperability of the information. Some attempts are present in the literature,

\footnotetext{
${ }^{1}$ Edilizia e opere di ingegneria civile - Gestione digitale dei processi informativi delle costruzioni
} 
mainly devoted on the integration of existing BIM platforms with other technical solutions. For example, in (Dore and Murphy, 2012) a two stage approach involves a 3D HBIM modelling stage and the integration of the 3D model into a 3D GIS for further management and analysis. The aim of this research is to bridge the gap between parametric CAD modelling and 3D GIS while using benefits from both systems to help document and analyze cultural heritage sites, providing an interoperable framework for modelling 3D geometries, semantics, topology and appearance properties. A wider discussion about this topic is reported in (Saygi and Remondino, 2013), a review of the current management approaches for architectural heritage information with BIM and GIS tools, illustrating up-to-date approaches and describing possible future research directions.

One of the technologies that can straightforwardly solve the issue of interoperability is the semantic web.

In fact, as several works in literature point out, not only in the $\mathrm{CH}$ domain, the use of Semantic-web standards, namely RDF and OWL, as formal knowledge representation language have several advantages (Costa and Madrazo, 2015); the main one is the possibility to flexibly mix BIM data and additional data created by operators via the data enrichment, and to represent multiple 3D models in the same computational space, thus being able to perform cross-model queries. Semantic technologies' advantage is exchanging information in a meaningful way, in a human readable format and with a minimum of human intervention. Due to the nature and large amount of data collected to implement a (H)BIM, semantic-web has demonstrated to fit with the existing standard format developed for such domain (Pauwels and Van Deursen, 2012). Closer to our approach HBIM related to the semantic web has been experienced in (Garagnani, 2015).

\section{WORKFLOW}

Following the need to make the data, organized in a BIM environment, usable for the different actors involved in the restoration phase, we have created a pipeline that takes advantage of BIM platforms and semantic-web technologies, enabling the user to query a repository composed by semantically structured and rich HBIM data. The presented pipeline follows the main steps listed below that, for clarity of explanation, are also reported in Figure 3:

- modelling an ontology with the main information needs for the domain of interest, providing a data structure that can be leveraged to inform the data-enrichment phase and, later, to meaningfully query the data;

- data enrichment, by creating a set of shared parameters reflecting the properties in our domain ontology;

- structuring data in a machine-readable format through a data conversion to represent the domain (ontology) and analyse data of specific buildings respectively; this step is mandatory to reuse the analysis data together with the 3D model, providing the end-user with a querying tool.

- development of a demonstrative data exploration web application based on the faceted browsing paradigm and allowing to exploit both structured metadata and $3 \mathrm{D}$ visualization.

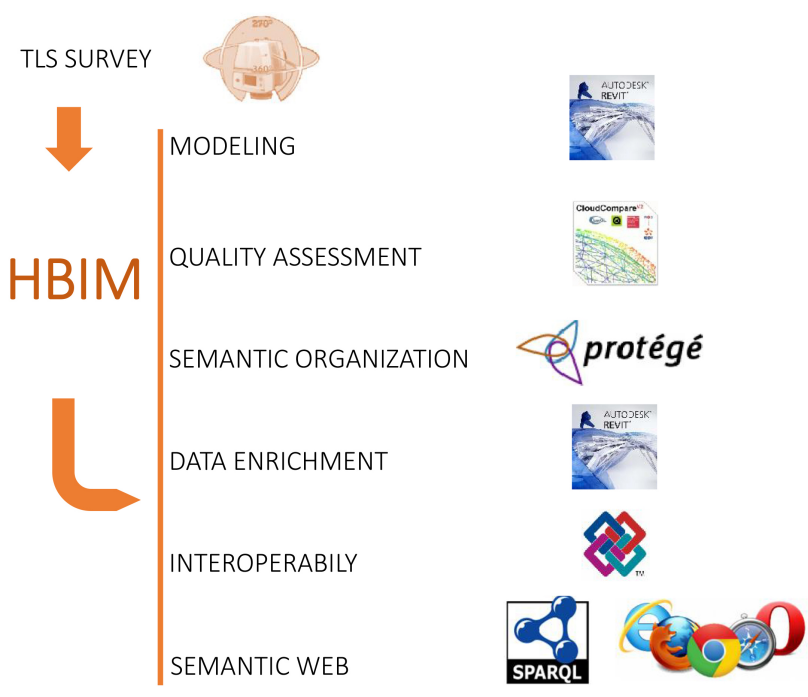

Figure 3. The procedure for obtaining HBIM devoted to restoration project: tools, steps and software.

\subsection{HBIM and ontology modelling}

The first step in the proposed workflow consists on managing, on the existing HBIM of the church, the main information related for the domain of interest. The data structure provided constitutes the backbone to inform the data-enrichment phase, as well as to perform advanced queries at the end of the process. In our case, we are interested in exploiting both the additional information related to the 3D model of the historical buildings, describing, for example, possible damages or recording the date of the last inspections of each architectural element (Figure 4).

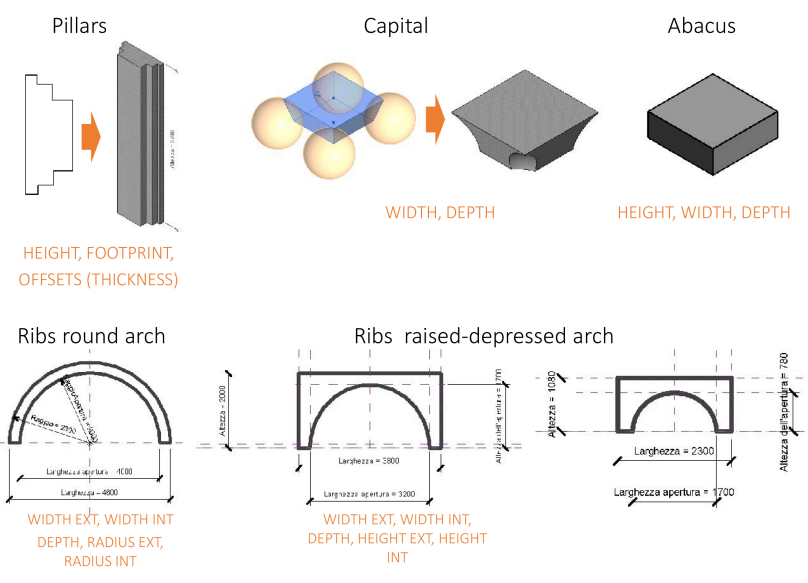

Figure 4. Parametric objects crated for the Santa Maria di Portonovo 3D Model in Revit.

The second stage of this procedure is the ontology for managing all information dividing both building and metadata in different layers with different depth. Since our final goal is to reuse the analysis data together with the spatial and 3D model, to provide querying and end-user browsing, we need to structure data in a machine-readable format. In line with the research trend, RDF (Resource Data Framework) and OWL (Ontology Web Language) were chosen in this research to represent the domain (ontology) and analysis data of specific buildings respectively. Specifically, we rely on the IFC-owl ontology to represent BIM data, and a custom ontology, to model additional domain data. Each 
Building Element can then be described by a set of properties hierarchically arranged in a taxonomy. For example, all the properties describing the architectural elements analysis results, e.g., damage type and damage morphology, with the related sub - properties of the property analysis results. The general overview of the taxonomy, related to the ontology is described in Figure 5.

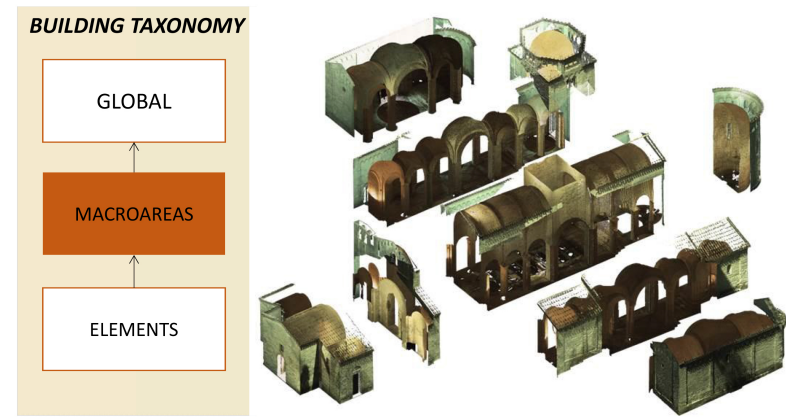

Figure 5. The building taxonomy at the intermediate level of macroareas. The ontology modelling follows the classical subdivision used in static analysis (macro-elements).

\subsection{Data enrichment}

While BIM data describing a 3D model in Resource Data Framework (RDF) can be obtained leveraging a standard Industry Foundation Classes (IFC) export available in most of the 3D modelling tool used in the HBIM community, providing users with a way of enriching the model with domain dependent data is less straightforward. Such a data enrichment step can be done at different times:

- Contextually with the 3D modelling. This is the approach we adopt and demonstrate in this paper by relying on IFC properties and their implementation in Revit, a widely used 3D modelling tool. This has the advantage of enabling users already familiar with Revit to perform data enrichment without the need of learning to use a new tool (Figure 6).

- Separately from the 3D modelling activity. Data enrichment could be done by annotating the IFC-OWL RDF data. While in some cases this could be the preferred method, as it allows to clearly separate the two activities and treat them independently, e.g. no need for going back to the 3D modelling tool if further data enrichment is needed, we do not address this methodology in the current paper, leaving it for future experimentations .

For our experiments we created a set of shared parameters reflecting the properties in our domain ontology. The data-enrichment process at first starts with the attribution of a label code that is necessary to uniquely identify instances. Starting from an already modelled building this phase was carried out by using an appropriate shared parameter. This expedient is necessary also to perform queries on specific elements. Later, in the process all elements must be singularly labelled and all the relative data sheets must be linked to them. It is so to be noted that in the Church object of this study, a great number of previous analysis and investigations were present, that allowed to built up a complex data enrichment for both the macro-elements (Figure 7) and the single elements (Figure 8), like for every wall, capital or column.

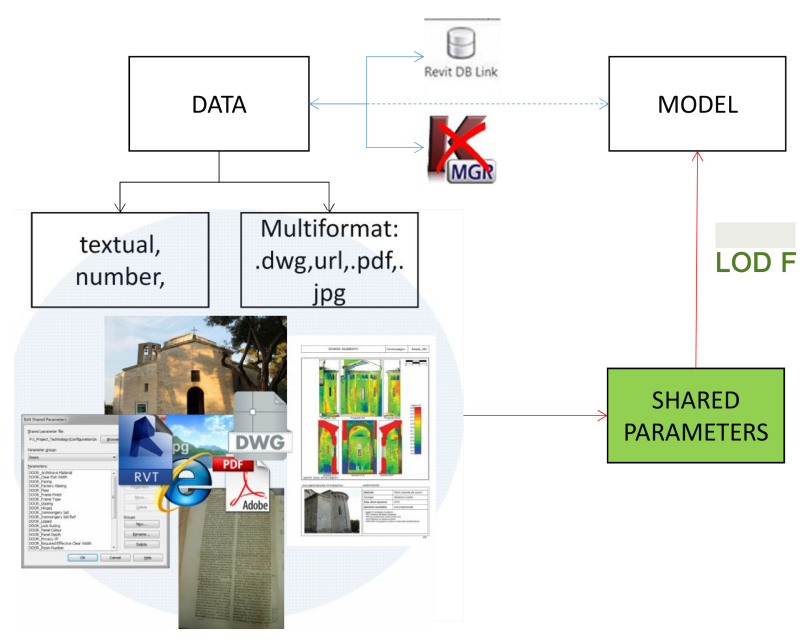

Figure 6. Data enrichment phase based on shared paramenters.
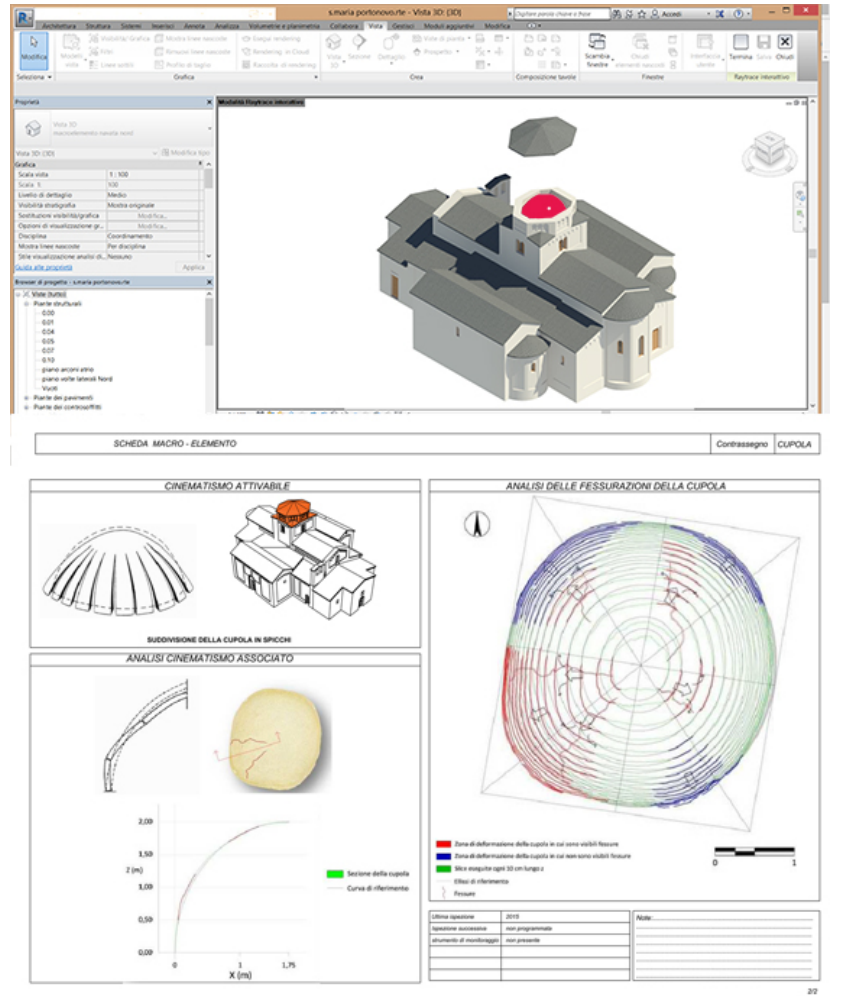

Figure 7. The data enrichment for a macroareas type. The connected data sheet contains static analysis based on comparison between TLS point cloud and geometric primitives.

\subsection{Data conversion for on-line exploration}

Once data enrichment has been done in Revit, the standard Revit data export functionality can be straightforwardly used to produce IFC data compliant with the EXPRESS schema. We then used the IFC-to-RDF conversion tool ${ }^{2}$ to obtain RDF data, including both BIM data and data enrichments. In this way, data enrichment can be captured by the IFC-OWL ontology by mapping IFC extensible properties. The resulting model has the advantage of being generic and flexible, but the drawback of being complex and requiring long SPARQL queries to extract relevant information. However, the SPARQL query language provides a

\footnotetext{
${ }^{2}$ https://github.com/mmlab/IFC-to-RDF-converter
} 

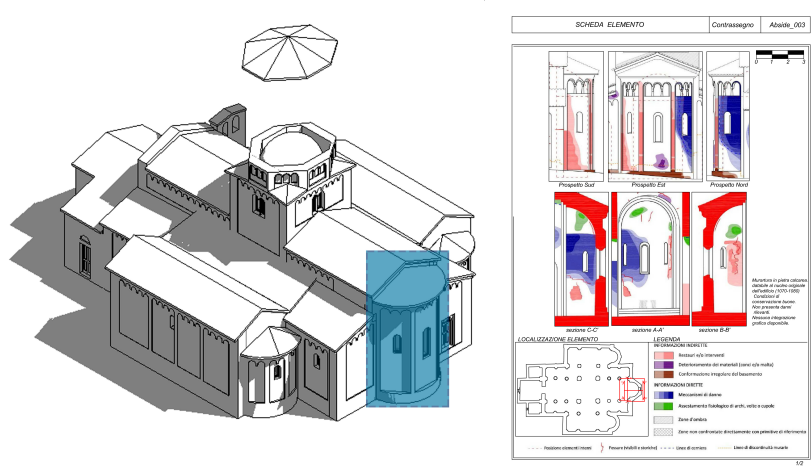

Figure 8. The data enrichment for an element. The linked file contains static analysis based on comparison between TLS point cloud and geometric primitives.

way of transforming the RDF graph, simplifying the data structure. It is so to be noted that the RDF data graph also contains all the information extracted from one or multiple BIM models. It is possible therefore to combine in a single query features like dimension of elements and placements and, in general, 3D model features, along with domain features, e.g. querying the system for all the damaged walls with last inspection before 2014, with no openings and below a given height.

Once all the data of interest are represented in RDF, we need to expose them to applications that wants to make use of such data. A variety of available RDF storage systems (called triplestores) can be used for this purpose. The choice of a particular triplestore is transparent to the applications using the data, as all triplestores expose the same standard SPARQL endpoint, based on simple HTTP calls. In our experiments we used the Sesame triplestore. While SPARQL provides a powerful, standardized way of querying the data, end-users need a more intuitive way of exploring the data. The major limitation is represented by the difficulty for a non-technician to write SPARQL queries and understand the domain ontology (Lee et al., 2016). On the other hand, querying data is not sufficient, as users need to actually see the architectural elements visually in the context of the 3D model. To overcome this problem, as a final step in our workflow, we developed a demonstrative data exploration web application based on the faceted browsing paradigm and allowing to exploit both structured metadata and 3D visualization. In the following section we demonstrate how such an application can be configured by a domain expert to reflect a given domain ontology, and used by an operator to query and explore the data.

\section{RESULTS}

Thanks to the pipeline of work described in the previous section, we are now allowed to query the resulting data (containing all the information added during the data enrichment phase) via the standard Semantic-web query language (SPARQL). However, the complexity of the IFC model, and specifically the pattern used to represent custom properties, makes writing such queries cumbersome. In order to, on one hand, make queries easier and, on the other hand, obtain data that conform to our domain ontology, we performed additional transformations on the RDF graph. To do so we used SPARQL construct queries as transformation rules to map from the IFC pattern to our domain ontology. Detailed discussion of such a transformation technique are out of the scope of this paper, interested readers can rely on the SPARQL spec- ifications to gain detailed technical understanding ${ }^{3}$. After the transformation is performed, the query can be done as follows: users can select a specific building or navigate across the architectural elements placed in different buildings, based on their IFC type or other domain specific features (Figure 9).
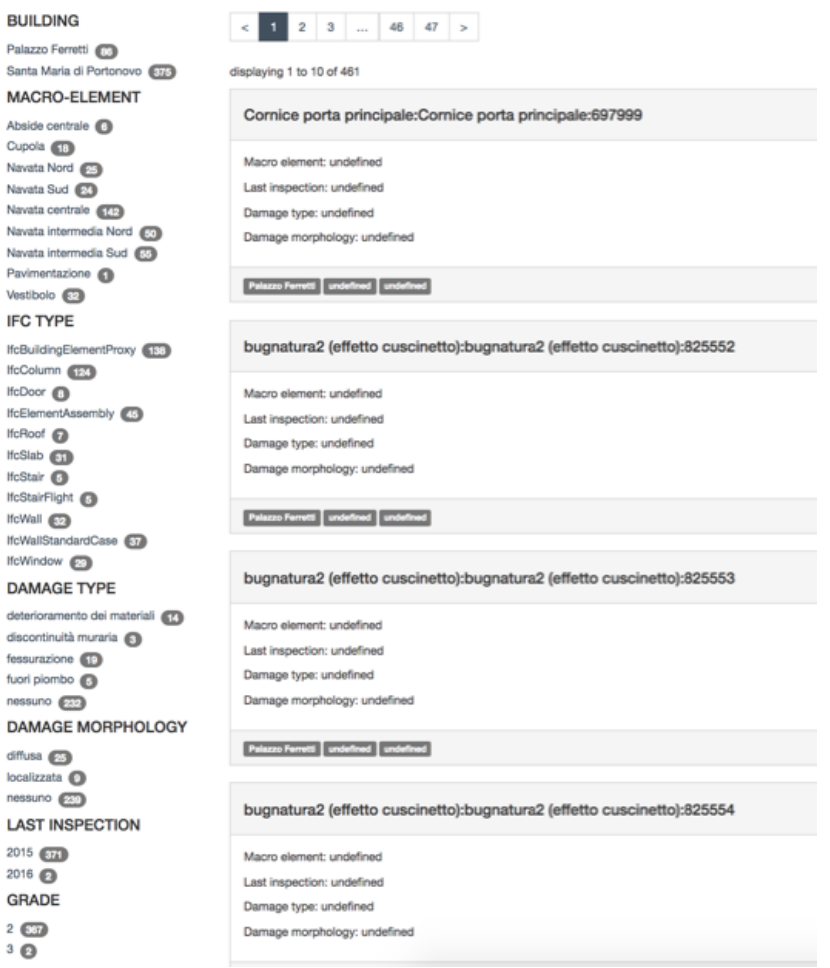

Figure 9. The faceted browser showing all the available filters.

Once users have filtered search results to individuate elements of interest, they immediately can view them in the 3D model. In this example, a user selected the Santa Maria di Portonovo building and filtered element of type Wall with last inspection on 2015. Such elements are shown in yellow in the 3D model, users can then further refine the model visualization by selecting and deselecting elements. Once a set of filters if set, available values in the facets (left menu) show the distribution of domain features across the selection. For example in this case she immediately sees how selected walls are distributed across macro-elements, that there are 4 damaged walls, etc. (Figure 10).

\section{DISCUSSION AND CONCLUSION}

The developed tool underpins several main aspects that is important to underline. It is completely based on widespread standards languages and on open source software; this make the tool completely detached from existing proprietary software like Revit. Further, it is worth to notice that the flexibility opens up this approach to future customization for different domains. In fact, facets can be easily customized according to the domain of the ontology that describes the BIM. This is not trivial, since every time one has to cope with domain specific information, architectural elements can be filtered accordingly and visualized in their 3D context within the browser. This can be very helpful especially in the case of monitoring the status of conservation of a historical building. The proposed solution in fact enables the practitioners to investigate over each single architectural component,

\footnotetext{
${ }^{3}$ https://www.w3.org/TR/rdf-sparql-query/
} 


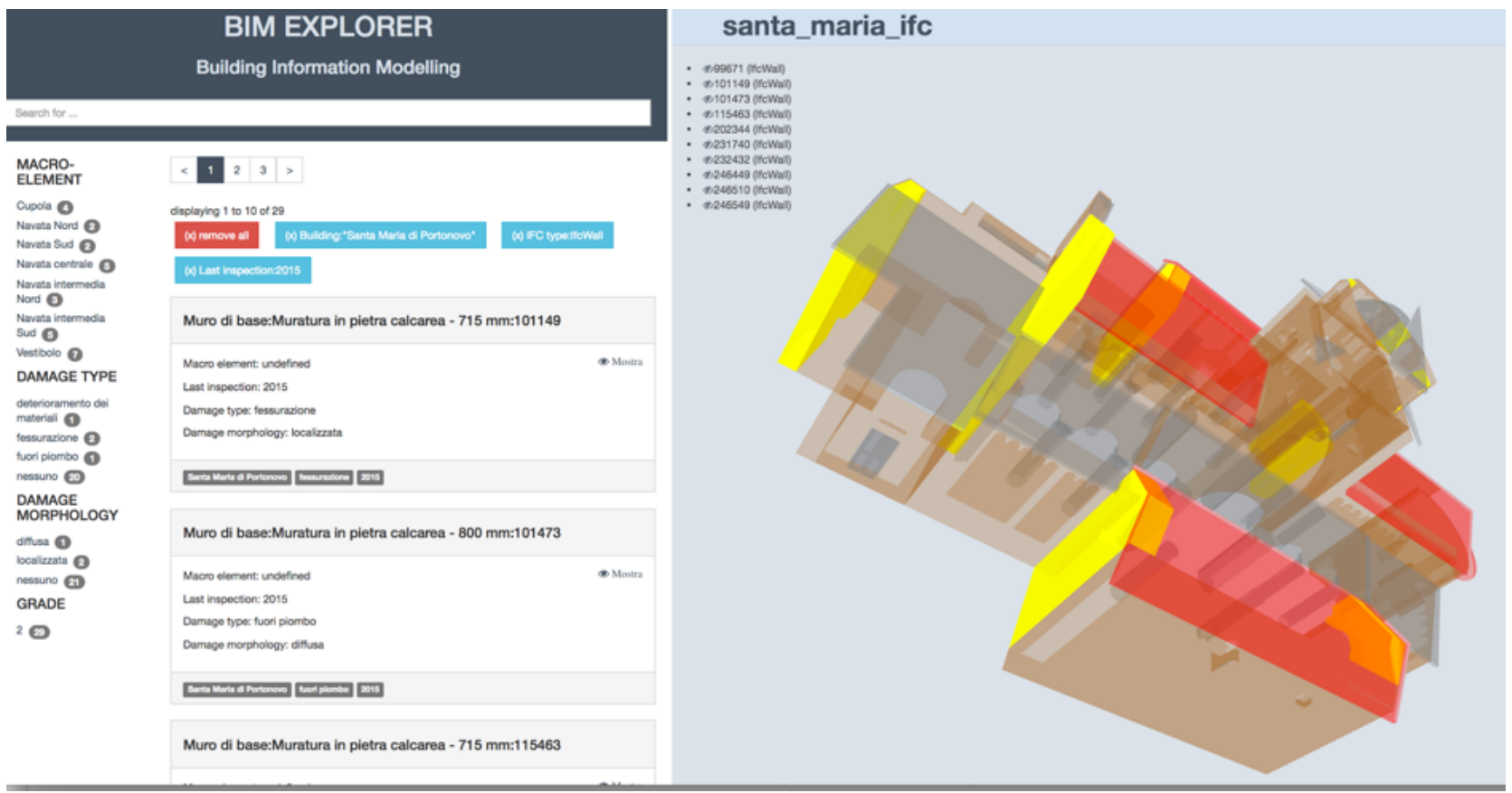

Figure 10. the visualization in 3D of the elements that have been searched through the faceted browser.

getting precise and reliable information related to the HBIM. Is it so to be noted that the BIMExplorer also contains all the information extracted from the enriched HBIM models, such as additional links. It is possible therefore to combine in a single query features like dimension of elements and placements and, in general, 3D model features, along with domain features, e.g. querying the system for all the damaged walls with last inspection before 2014, with no openings and below a given height.

We can summarize that the work described opens a new scenario for the exploitation of enriched (H)BIM. Up to know, making precise and advanced queries over semantically structured historical buildings models is not allowed by nowadays BIM tools. Thanks to the BIMexplorer it is possible to guarantee a complete interoperability with IFC/RDF format, besides allowing to discover the knowledge among experts and non experts users. The migration of data from a standard HBIM to the semantic-web tool, in which users can browse the model filtering properties and viewing the $3 \mathrm{D}$, represent the main novelty of this work. While the data enrichment phase paves the way for the enhancement of the informative nature of the model, the possibility to interact with those data outside the BIM environment is valuable. As a matter of fact, the data displayed could be of different typologies and they can refer:to 3D models (or portions, drawing and multimedia contents The workflow also showed how it is possible to move from geometric HBIM representation towards web 3D objects management, supporting enhanced comprehension of the single elements within the model information of the overall building organism, and allowing the connection to descriptive thematic database (constructive technologies, elements' abacus), in a logic of semantic content models. The main weak point of the research, that also depicts our future works, is that actually the ontology modeling phase and the data enrichment phase are detached. Indeed, at the present stage, the ontology implementation is not readable in the Revit model and in the IFC format. In this light, it could be useful also to automate the link between IFC/RDF format and OWL taxonomy.

For the specific domain of this article, we can say that restorers could take advantage from this tool, since all the information related to the presence of damages (and their nature), the last year of inspection, the link to pictures and 2D modelling can be easily obtained with a more friendly and intuitive tool Our approach, if performed through a widespread standardization, will be a key element for the diffusion of HBIM in professional practices, but it could lead to interesting results in the field of representation and information technology research. In this framework, the present research answers to the following relevant topics: definition of the different categories of items from a reality-based survey and their semantic organization in HBIM; connection between model and information considering each single item like a large repository; management of items/repositories of information in web libraries devoted to historical buildings. In this way, the planned maintenance or the restoration project became collaborative and interactive, optimizing the whole process of HBIM data collection.

\section{ACKNOWLEDGEMENTS}

The authors would acknowledge the FAI (Italian Ambiental Found) and the Superintendence for Architectural Heritage and Landscape of the Marche Region for allowing the acquisition of the Church of S. Maria at Portonovo. The data enrichment phase was carried out by Andrea Carassai, in his degree thesis supervised by Ramona Quattrini, Eva S. Malinverni and Roberto Pierdicca. The development of the web tools was possible thanks to the contribution of Manolo Micozzi. The authors also want to thank Paolo Clini, Enrico Quagliarini and Mirco Ripanti for sharing drawings and analysis used in the data HBIM collection.

\section{REFERENCES}

Achille, C., Fassi, F. and Fregonese, L., 2012. 4 years history: From $2 \mathrm{~d}$ to bim for ch: The main spire on milan cathedral. In: Virtual Systems and Multimedia (VSMM), 2012 18th International Conference on, IEEE, pp. 377-382. 
Apollonio, F. I., Gaiani, M. and Sun, Z., 2012. Bim-based modeling and data enrichment of classical architectural buildings. SCIRES-IT-SCIentific RESearch and Information Technology 2(2), pp. 41-62.

Apollonio, F. I., Gaiani, M. and Sun, Z., 2016. A reality integrated bim for architectural heritage conservation. Handbook of Research on Emerging Technologies for Architectural and Archaeological Heritage p. 31 .

Biagini, C., Capone, P., Donato, V. and Facchini, N., 2016. Towards the bim implementation for historical building restoration sites. Automation in Construction 71, pp. 74-86.

Centofanti, M. and Brusaporci, S., 2012. Architectural 3d modeling in historical buildings knowledge and restoration processes. Less More architecture design landscape. Aversa (Italia), Capri (Italia), 21 magggio 2012-2 giugno 2012, NAPOLI: La Scuola di Pitagora, ISBN.

Costa, G. and Madrazo, L., 2015. Connecting building component catalogues with bim models using semantic technologies: an application for precast concrete components. Automation in Construction 57, pp. 239-248.

Dore, C. and Murphy, M., 2012. Integration of historic building information modeling (hbim) and $3 \mathrm{~d}$ gis for recording and managing cultural heritage sites. In: Virtual Systems and Multimedia (VSMM), 2012 18th International Conference on, IEEE, pp. 369376.

Felicetti, A. and Lorenzini, M., 2011. Metadata and tools for integration and preservation of cultural heritage $3 \mathrm{~d}$ information. Geoinformatics FCE CTU 6, pp. 118-124.

Garagnani, S., 2015. Semantic representation of accurate surveys for the cultural heritage: Bim applied to the existing domain. Emerging Digital Tools for Architectural Surveying, Modeling, and Representation pp. 292-310.

Lee, D.-Y., Chi, H.-1., Wang, J., Wang, X. and Park, C.-S., 2016. A linked data system framework for sharing construction defect information using ontologies and bim environments. Automation in Construction 68, pp. 102-113.

Li, Y., Dong, K. and Li, G. F., 2014. The application of bim in the restoration of historical buildings. In: Applied Mechanics and Materials, Vol. 638, Trans Tech Publ, pp. 1627-1635.

Logothetis, S., Delinasiou, A. and Stylianidis, E., 2015. Building information modelling for cultural heritage: a review. ISPRS Annals of the Photogrammetry, Remote Sensing and Spatial Information Sciences 2(5), pp. 177.

Ma, Y., Hsu, C., Lin, M., Tsai, Z. and Chen, J., 2015. Parametric workflow (bim) for the repair construction of traditional historic architecture in taiwan. The International Archives of Photogrammetry, Remote Sensing and Spatial Information Sciences 40(5), pp. 315 .

Pauwels, P. and Van Deursen, D., 2012. Ifc/rdf: adaptation, aggregation and enrichment. In: First international workshop on linked data in architecture and construction (LDAC 2012).

Quattrini, R., Malinverni, E., Clini, P., Nespeca, R. and Orlietti, E., 2015. From tls to hbim. high quality semantically-aware $3 \mathrm{~d}$ modeling of complex architecture. The International Archives of Photogrammetry, Remote Sensing and Spatial Information Sciences 40(5), pp. 367.

Saygi, G. and Remondino, F., 2013. Management of architectural heritage information in bim and gis: State-of-the-art and future perspectives. International Journal of Heritage in the Digital Era 2(4), pp. 695-713.
Volk, R., Stengel, J. and Schultmann, F., 2014. Building information modeling (bim) for existing buildingsliterature review and future needs. Automation in construction 38, pp. 109-127. 